\title{
N ANTECEDENTE IMPRESCINDIBLE DEL VALLE DE LOS CAÍDOS: LA APORTACIÓN DE MANUEL ABRIL EN 1939
}

INÉS ESCUDERO GRUBER

Universidad de Zaragoza inescu@unizar.es

\begin{abstract}
Resumen: En abril de 1939 veía la luz un artículo firmado por el crítico de arte Manuel Abril en el que abogaba por la existencia de un monumento para celebrar la victoria de la guerra y honrar a sus caídos. En esa misma fecha se dictó el decreto que disponía la erección de un monumento para perpetuar el recuerdo a los muertos en la Cruzada de Liberación, lo que supuso el primer paso para la construcción del Valle de los Caídos. Las coincidencias conceptuales y plásticas entre ambos monumentos, el propuesto y el ejecutado, son más que notorias. Es propósito de este trabajo ponerlas de manifiesto dentro del contexto histórico y conceder a la aportación de Abril la categoría de antecedente del monumental Valle de los Caídos.
\end{abstract}

Palabras clave: Valle de los Caídos / Manuel Abril / antecedente / monumentos a los caídos / arquitectura franquista.

\section{AN ESSENTIAL ANTECEDENT OF THE SPANISH VALLEY OF THE FALLEN: MANUEL ABRIL'S CONTRIBUTION ON 1939}

Abstract: On April 1939, art critic Manuel Abril published an article where he claimed the need for a monument through which Spanish people could celebrate the victory of the war and honor its fallen soldiers. On that same date, it was issued a decree arranging the erection of a monument destined to perpetuate the memory of those who died in the Crusade of Liberation, which was the first step towards the construction of the the site known as Valley of the Fallen. The conceptual and formal coincidences of both monuments, the one proposed and the one actually built, are more than notorious. The purpose of this work is to bring them to knowledge within the historical context and to consider Abril's contribution as a key antecedent for the monument called Valley of the Fallen.

Keywords: Valley of the Fallen / Manuel Abril / antecedent / monuments to the fallen / Francoist architecture.

\section{El nacimiento de la idea}

El monumento del Valle de los Caídos [fig. 1] ha sido objeto de múltiples estudios y artículos de opinión en numerosas ocasiones a lo largo de los años. Recientemente el sitio de Cuelgamuros ha vuelto a situarse en el foco de atención debido a la polémica e internacionalmente mediática exhumación de los restos de Francisco Franco. El nombre del dictador ha estado siempre vinculado a la monumental construcción sita en la madrileña sierra de
Guadarrama, y no solo porque se le diera sepultura allí o por tratarse de un emblemático monumento ligado al régimen, sino por considerar a Franco el artífice del proyecto, la persona que concibió ese inmenso hito conmemorativo y le otorgó su sentido y razón de ser.

La historia comenzaba así: "El mismo día de la Victoria, primero de abril de mil novecientos treinta y nueve, en el que el último Parte Oficial del Cuartel General del Generalísimo de los Ejércitos Na-

* Fecha de recepción: 15 de abril de 2020 / Fecha de aceptación: 23 de noviembre de 2020. 


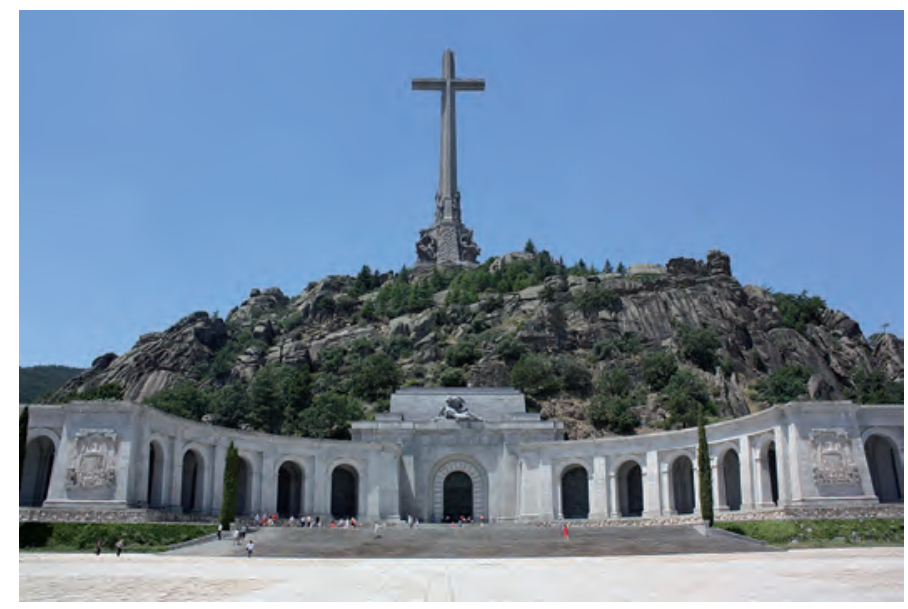

Fig. 1. Valle de los Caídos, 1940-1958. Cuelgamuros, Madrid.

cionales anunciaba que la Guerra había terminado, se dictó un Decreto disponiendo la erección de un magno monumento destinado a perpetuar la memoria de los Caídos en la Cruzada de Liberación, para honra de quienes dieron sus vidas por Dios y por la Patria y para ejemplo de las generaciones venideras". ' Son las primeras palabras de la disposición por la que se establecía en 1957 la Fundación de la Santa Cruz del Valle de los Caídos, momento en que ya se acercaba el final de los casi interminables trabajos de construcción. La referencia al decreto de 1939 y al destino explícito de ese "magno monumento" no dejan lugar a dudas: el memorial se concibió como tributo a quienes cayeron en el lado de Franco.

Bien es cierto que, si continuamos la lectura de la orden del 57, encontraremos un intento -endeble, a nuestro juicio - por ampliar el homenaje del monumento a todos los muertos, lo que se hacía en nombre de su carácter cristiano, que "ha de ir siem- pre acompañado del sentimiento de perdón", unido a las circunstancias de paz y a la unidad de los españoles, tal y como se indicaba en el texto. ${ }^{2}$

Franco imaginó un monumento colosal cuya magnitud fuese digna de la gesta que quería inmortalizar para que aleccionara a las generaciones venideras, y así lo expuso en el decreto del primer aniversario de la victoria. La dimensión de su Cruzada y "la trascendencia que ha tenido para el futuro de España esta epopeya" ${ }^{3}$ demandaban una construcción que emulara la grandeza de los antiguos y que de ninguna manera podía encontrar una justa representación en los sencillos monumentos con los que solía conmemorarse el triunfo en las ciudades.

El dictador hizo de esta gran empresa su particular obsesión, supervisando personalmente cada detaIle. De hecho, todo lo relacionado con el Valle dependió directamente de la Presidencia del Gobierno. ${ }^{4}$ Fue tal su implicación que realizó varias salidas para localizar el emplazamiento perfecto (su búsqueda concluyó al encontrar el imponente risco de la Nava en la zona conocida como Cuelgamuros, en las inmediaciones de El Escorial al que tanta devoción profesaba), y hasta esbozó los croquis de los planos con el diseño que atisbaba. Las declaraciones del primer arquitecto que estuvo al frente del proyecto así lo corroboran. Pedro Muguruza, a la sazón Director General de Arquitectura, ${ }^{5}$ aseguraba que a pesar de haber trazado él los planos, fue el Jefe de Estado quien había planteado las directrices a seguir. ${ }^{6} \mathrm{~A}$ estas alturas es ya sabido el interés del caudillo por la arquitectura, además de su afición a la pintura y su destreza con el dibujo. Un interés que iba más allá de su consideración política y propagandística como "arquitecto de la patria", responsable de la grandiosa reconstrucción de una aquejada España tanto en el plano urbanístico como en el moral.

\footnotetext{
1 España. Decreto-ley de 23 de agosto de 1957 por el que se establece la Fundación de la Santa Cruz del Valle de los Caídos. Boletín Oficial del Estado, 5-9-1957, núm. 226, p. 834-835.

${ }^{2}$ Lo cual no estuvo exento de ciertos desencuentros, como el protagonizado por el padre jesuita E. Guerrero, quien en un artículo publicado en la revista Razón y Fe al año siguiente, sostenía que el monumento debía dedicarse en exclusiva a los caídos en el bando vencedor. GUERRERO, E., 1958.

${ }^{3}$ España. Decreto de 1 de abril de 1940 disponiendo se alcen Basílica, Monasterio y Cuartel de Juventudes, en la finca situada en las vertientes de la Sierra del Guadarrama (El Escorial), conocida por Cuelga-muros, para perpetuar la memoria de los caídos en nuestra Gloriosa Cruzada. Boletín Oficial del Estado, 2-4-1940, núm. 93, p. 2240.

4 LLORENTE HERNÁNDEZ, Ángel, 1995, p. 105.

${ }^{5}$ El arquitecto vasco fue el primero en ocupar este puesto desde la creación del servicio en 1939, y parecía lógico y coherente encomendarle la dirección del monumento cuya inminente construcción se iniciaría al año siguiente.

${ }^{6}$ OLMEDA NICOLÁS, Fernando, 2009, p. 36.
} 


\section{La política arquitectónica del franquismo}

La práctica de la arquitectura en los años que siguieron al conflicto bélico se entendió como una actividad política vinculada al Estado. Es más, la Dirección General de Arquitectura ocupada en primera instancia por Muguruza estaba adscrita al Ministerio de la Gobernación. Desde esa Dirección se puso en marcha un proceso de depuración de arquitectos que atendía a cuestiones "indisimuladamente políticas o sociales" y no tanto a las profesionales. ${ }^{7}$ El proceso fue además apremiado por la urgente demanda de arquitectos, "una profesión cuyas actividades son hoy excepcionalmente reclamadas con premura en su función reconstructora", rezaba una orden de la citada Dirección. ${ }^{8}$

La necesaria reconstrucción de España tenía asociada además una dimensión espiritual, como anotábamos antes. Franco se alzaba como el gran reparador de las ruinas físicas y morales que habían soportado tres años de violencia. Bien conocida es la utilización propagandística por parte del régimen de estos vestigios como elementos recurrentes en la política reconstructora, y no solo en términos arquitectónicos: la ruina como símbolo del renacer de un nuevo orden. Todo un ideario que aunaba Arquitectura y Estado.

Ese potente binomio precisaba de una puesta en escena sólida, segura y también reconocible. Era imprescindible construirse una imagen propia, un estilo que pudiera llamarse franquista y a través del que poder asociar de un vistazo el progreso con el nuevo Estado, responsable del mismo.

Los teóricos abogaban por un estilo de corte clásico, imperial, con rasgos tomados de la propia tradición española. Tuvieron como referente la arquitectura filipina, con el monasterio del Escorial como ejemplo a seguir, pues el estilo herreriano fue muy alabado e imitado. Igualmente observaron la actuación de otros países, especialmente Alemania, donde la arquitectura era igualmente considerada un arte superior. Hitler, como Franco, sentía pasión por esta disciplina que resultaba ser, ade- más, la forma más elocuente de reflejar el poderío del III Reich.

Sin embargo, encontrar un estilo propio no era tarea fácil. Las tentativas por definir los rasgos estilísticos de la arquitectura oficial quedaron reducidas precisamente a eso: a intentos más teóricos que prácticos en una búsqueda insistente pero frustrada. ${ }^{9}$ Principalmente se debe atribuir este hecho a la acusada limitación de recursos que, paradójicamente, fue una de las causas definitorias de estilo: si los edificios carecían de esculturas monumentales, no era tanto debido a una apuesta por la sobriedad clasicista como por la escasez de materiales.

En este contexto debemos situar la construcción del Monumento Nacional a los Caídos, como se le bautizó oficialmente. Es decir, una obra arquitectónica de importancia incomparable para el caudiIlo, que pretendió ser la expresión arquitectónica del régimen plasmando la victoria que posibilitó la tabula rasa sobre la que levantar el nuevo Estado. Una obra, además, de dimensiones descomunales, proporcionales a su relevante significado político, con todas las dificultades que ello conllevaba en cuanto a recursos humanos y materiales.

\section{De la quimera a la realidad: la fabricación de una tipología monumental en recuerdo a los caídos}

Cuando se habla de los antecedentes del Monumento Nacional a los Caídos se menciona el artículo publicado en Vértice, la revista Nacional de Falange Española Tradicionalista y de las JONS, con el título "Sueño arquitectónico para una exaltación nacional", firmado por el escultor Manuel Álvarez Laviada, el arquitecto Luis Moya y el militar Gonzalo Serrano, vizconde de Uzqueta. ${ }^{10} \mathrm{El}$ ejercicio quimérico se presentaba a través de varios dibujos y un plano que mostraban una interesante construcción de tipo visionario al estilo de la arquitectura revolucionaria del neoclasicismo, concebida en plena guerra civil en territorio hostil ("el Madrid rojo" como se lee en el texto) [fig. 2]. ${ }^{11}$ Los autores, animados en ese momento por una ansia-

\footnotetext{
7 GARCÍA-GUTIÉRREZ MOSTEIRO, Javier, 2008, p. 355.

${ }^{8}$ Orden de 24 de febrero de 1940 de la Dirección General de Arquitectura. Extraído de GARCÍA-GUTIÉRREZ MOSTEIRO, Javier, 2008, p. 356

9 LLORENTE HERNÁNDEZ, Ángel, 1995, p. 80.

10 LAVIADA, Manuel; MOYA, Luis; SERRANO, Gonzalo, 1940. Haciendo referencia precisamente a ese artículo arranca Daniel Sueiro su libro La verdadera historia del Valle de los Caídos, bajo el epígrafe "La idea y su puesta en marcha" (SUEIRO, Daniel, 1976 , p. 9), volumen que constituye un referente sobre la historia del monumento y que ha sido recientemente reeditado (Editorial TÉBAR FLORES, 2019).

11 Dos de los dibujos originales del arquitecto Luis Moya, concretamente el de la vista exterior de la pirámide y vista del lado norte, y el de la axonometría seccionada de la pirámide, ambos reproducidos en el artículo de Vértice (puede verse el segundo en la parte inferior derecha de la figura 2), se exhibieron en la exposición Campo cerrado. Arte y poder en la posguerra española, 1939-1953, celebrada en el Museo Nacional Centro de Arte Reina Sofía en 2016, fundamental para comprender la producción
} 


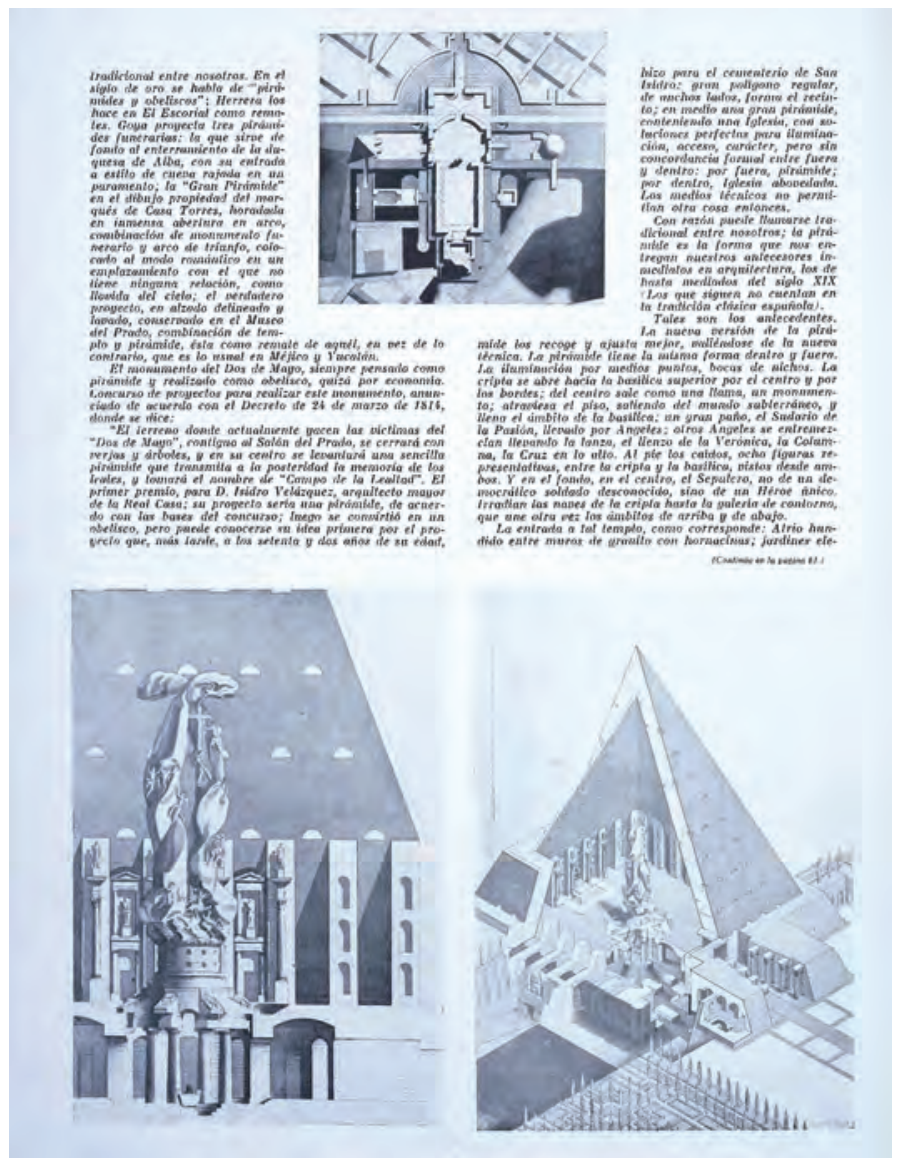

Fig. 2. Página del artículo "Sueño arquitectónico para una exaltación nacional” firmado por Manuel A. Laviada, Luis Moya y el vizconde de Uzqueta, publicado en el núm. 36 de la revista Vértice (septiembre de 1940).

da victoria, diseñaron un monumento que aunara tres ideas clave: "una exaltación fúnebre, nacida de lo que sucedía alrededor y de lo que amenazaba; la idea triunfal, que producía lo que se oía y lo que se esperaba; una forma militar, reacción contra la indisciplina ambiente". ${ }^{12}$ En verdad, ese trío nuclear -el fúnebre, triunfal y militar, al que se une además y casi de forma inherente el componente religioso- es el nexo más evidente entre esta ensoñación y la realidad que se materializó en torno al risco de la Nava. Se trata de un vínculo intencional y no tanto formal, una "premonición del Valle de los Caídos", concede Antonio Bonet Correa, ${ }^{13}$ que incorporaba elementos emparentados con la tradición clasicista de tiempos pasados y que, como hemos señalado, fueron la base sobre la que se quiso construir el estilo arquitectónico propio de la dictadura franquista. Con todo, este mismo autor calificaba el proyecto como la obra más vanguardista de las publicadas en Vértice junto con los dibujos de José Caballero. ${ }^{14}$

El proyecto imaginado por Moya, Laviada y el vizconde de Uzqueta se remataba con una pirámide, forma de ancestral tradición funeraria, no solo empleada como mausoleo sino también como homenaje a los caídos. Incluso Goya ideó una pirámide en su diseño de monumento a los caídos en la Guerra de la Independencia, y no es casual que los autores del proyecto publicado en Vértice optaran por introducirla en sus planos y hasta situarla como elemento de coronación del templo, igual que Goya. De hecho, indicaron explícitamente su vinculación con la propuesta del genio aragonés. ${ }^{15}$

Sin embargo, la pirámide no terminaría cuajando en los memoriales levantados en los años de la dictadura. ${ }^{16} \mathrm{Ni}$ siquiera lo haría el obelisco, que podríamos considerar una pirámide estilizada y que, por cierto, fue la forma que domina el monumento definitivo a los héroes del dos de mayo en la plaza de la Lealtad de Madrid, junto al Paseo del Prado, en nada emparentado con el proyectado por Goya. Sobre ambas figuras pesaba el inconveniente del paganismo y, en un Estado confesional en el que el catolicismo jugaba un importante papel, eso suponía un problema no precisamente pequeño. En ese aspecto, es interesante recuperar las conclusiones del informe del jefe del Departamento de Plástica del Servicio Nacional de Propaganda rechazando una propuesta de proyecto conmemorativo en los albores de la dictadura: "Este proyecto no puede ser autorizado pues se trata en él de un simple Obelisco, forma arquitectónica que creemos poco oportuna para conmemorar el recuerdo de nues-

artística en sus diversas manifestaciones durante el primer franquismo. JIMÉNEZ-BLANCO, Maa Dolores (com.), 2016, p. 146-148 y 337-338.

12 JIMÉNEZ-BLANCO, Ma Dolores (com.), 2016, p. 7.

13 BONET CORREA, Antonio, 1981, p. 33.

14 BONET CORREA, Antonio, 1981, p. 33.

${ }^{15}$ LAVIADA, Manuel; MOYA, Luis; SERRANO, Gonzalo, 1940, p. 7.

${ }^{16}$ Llorente dio noticia de un solo proyecto de monumento con forma de pirámide en la localidad riojana de Igea. LLORENTE HERNÁNDEZ, Ángel, 1992, p. 178. 
tros caídos. En efecto, el obelisco terminado en pirámide, lo mismo que esta, es un símbolo de la llama del fuego que todo lo consume, y por ello un símbolo puramente pagano de la muerte, que no encierra en sí ninguna idea de Redención, de Sacrificio, ni de vida Eterna". ${ }^{17}$

Este tipo de informes se expedían desde el Servicio Nacional de Propaganda, que debía emitir juicio favorable y autorizar expresamente la construcción de cada propuesta, ostentando así el control sobre todas las iniciativas conmemorativas. El primer paso en este sentido fue la creación en 1938 de la Comisión de Estilo en las Conmemoraciones de la Patria, con la intención de centralizar las decisiones relativas a la confección de todo tipo de elementos conmemorativos, desde inscripciones a grandes monumentos. La iniciativa fue ampliándose y concretándose legislativamente al terminar la guerra, como indica la orden del Ministerio de la Gobernación, desde donde se aspiraba a "dar unidad de estilo y de sentido a la perpetuación por monumentos de los hechos y personas de la historia de España". ${ }^{18} \mathrm{Se}$ quiso incluso elaborar un catálogo de modelos que sirvieran de referencia, en respuesta a la gran cantidad de memoriales que se proyectaba levantar. ${ }^{19}$ Fue esta rígida normativa reguladora la que propició que la mayoría de ellos resultaran tediosamente parecidos, constriñendo así la creatividad de los diseñadore ${ }^{20}$ y desembocando en la consolidación, en los años 40, de un prototipo monumental en recuerdo de los caídos, cuyo esquema básico incorporaba una cruz situada sobre una estructura a modo de podio. La cruz sería el elemento preferido para estos memoriales, convirtiéndose en el signo destinado a "concretar la alegría por la victoria". ${ }^{21}$ No en vano, el antes aludido informe concluía añadiendo que "A nuestros Caídos creemos que no se les debe conmemorar más que con la cruz". ${ }^{22}$

Por otra parte, y al mismo tiempo, esta censura contribuía a lograr una institucionalización del culto a la muerte, ${ }^{23}$ entrelazando el ritual sagrado con las ideas políticas y tejiendo un sólido corpus para el establecimiento de la doctrina del nacionalca- tolicismo. "Este exacerbado culto político nacionalista, y profano en algunas de sus manifestaciones, ocupó el espacio público, irrumpió en los lugares sagrados y se sirvió de los símbolos, los ritos y las celebraciones litúrgicas en el recuerdo de los 'caídos'", señala Sevillano-Calero. $^{24}$

De este modo, la renacida España se abarrotó de cruces. Por poner un ejemplo de estos modelos, firmado además por el autor de la sugerente ensoñación arquitectónica quien, evidentemente, también debió ceñirse a los cánones, citaremos el Monumento a los Héroes y los Mártires de Nuestra Gloriosa Cruzada de Zaragoza. Luis Moya realizó este proyecto en 1943 junto con los arquitectos Ramiro Moya (su hermano), Enrique Huidobro y el escultor Manuel Álvarez Laviada. Su diseño para la capital aragonesa incorporaba una cruz de notable tamaño colocada sobre una estructura clasicista a la manera de altar, y se completaba con otros elementos, como un frente mural que actuaba de cerramiento. No obstante, debieron introducir algunas modificaciones al diseño original, resultando en un monumento más sencillo, terminado un decenio después. ${ }^{25}$

Sin duda mucho más ambiciosa fue la propuesta con la que, de nuevo, Luis Moya y Enrique Huidobro, esta vez acompañados de Manuel Thomas, concurrieron al concurso de anteproyectos para una gran cruz monumental que convocó ese mismo año 1943 el Patronato del Monumento Nacional para seleccionar el remate del grandioso conjunto arquitectónico [fig. 3]. Una propuesta que no queremos dejar de mencionar, teniendo en cuenta su destino. Diseñaron una imponente cruz de proporciones sobrehumanas apeada sobre una estructura que alojaba un altar, y arropada por un viaducto que terminaba con otras dos cruces a los lados ya reducidas a la escala del hombre, así como el acceso frontal formado por una escalinata de doble ramal en algunos tramos. Los trabajos escultóricos del conjunto, como las figuras de los nichos dispuestos en el vástago de la cruz o las que coro-

\footnotetext{
17 Extraído de LLORENTE HERNÁNDEZ, Ángel, 1992, p. 178.

18 España. Orden de 7 de agosto de 1939 disponiendo queden supeditados a la aprobación de este Ministerio toda iniciativa de monumentos en general. Boletín Oficial del Estado, 22-8-1939, núm. 234, p. 4614.

19 LLORENTE HERNÁNDEZ, Ángel, 1995, p. 277-278.

20 LLORENTE HERNÁNDEZ, Ángel, 1995, p. 299.

21 Citado en OLMEDA NICOLÁS, Fernando, 2009, p. 54.

22 Extraido de LLORENTE HERNÁNDEZ, Ángel, 1992, p. 178.

${ }^{23}$ Sobre este asunto véase SEVILLANO-CALERO, Francisco, 2017.

24 SEVILLANO-CALERO, Francisco, 2017, p. 615

25 VÁZQUEZ ASTORGA, Mónica, 2006, p. 304.
} 


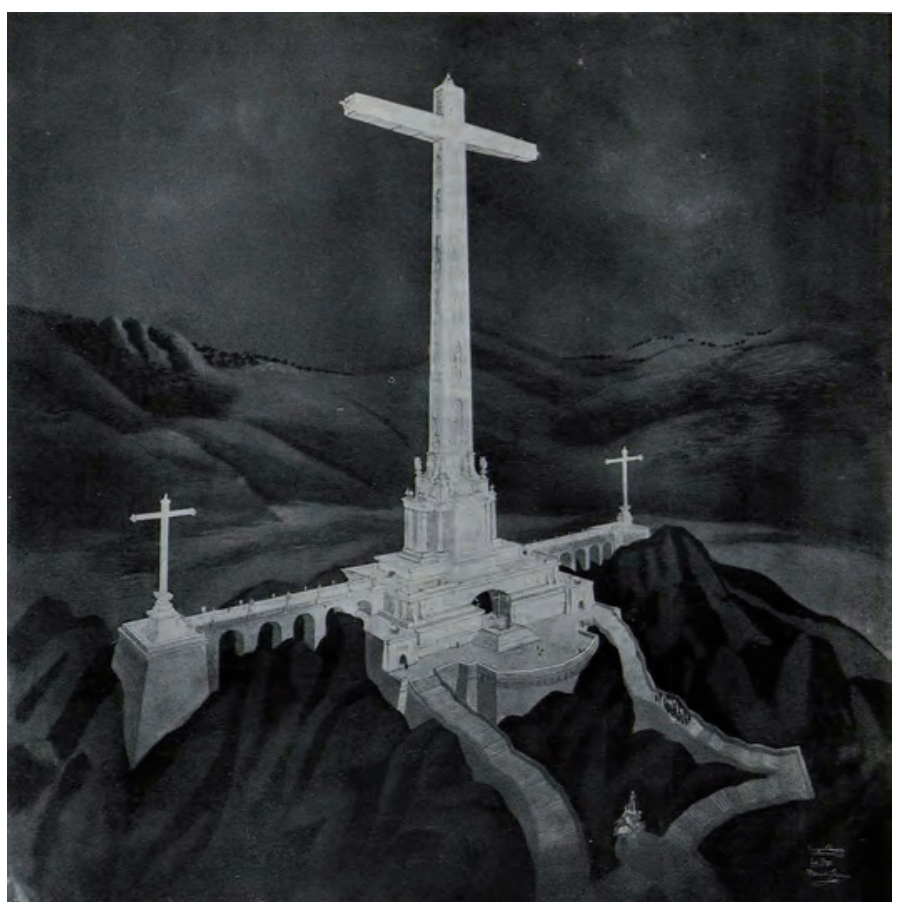

Fig. 3. Enrique Huidobro, Luis Moya y Manuel Thomas, Proyecto de una cruz monumental para el Monumento Nacional a los Caídos, 1943, publicado en el núm. 18-19 de la Revista Nacional de Arquitectura (junio-julio de 1943).

nan el viaducto, fueron diseñados por Álvarez Laviada. ${ }^{26} Y$ se alzaron con el primer premio, bien que finalmente no fuera su cruz la que se erigió.

En definitiva, esta tipología con la cruz como estandarte servía tanto para el recuerdo a los muertos como para la exaltación de la victoria. $Y$ es que el fuerte simbolismo cristiano de la cruz encajaba por su sentido fúnebre y luctuoso en el cometido de honrar a las víctimas (mártires y héroes), pero asimismo resultaba válido por representar el triunfo de Cristo sobre la muerte, traducido en la conquista y liberación perpetradas por Franco ante la amenaza que, en su opinión, se cernía sobre España; sufrimiento, por otra parte, equiparable al sa- crificio de Jesús de Nazaret en el madero. Esto es, la cruz como origen y justificación de la Cruzada de Liberación, pero también como símbolo pacificador que acoge y redime una vez terminado el conflicto. El principio y el fin, como el alfa y omega del crismón.

\section{Una interesante y temprana propuesta: el monumento pensado por Manuel Abril}

El crítico de arte Manuel Abril (Madrid, 1884-1943) ya vislumbró una cruz como la forma más acertada para celebrar e inmortalizar el triunfo bélico de las tropas de Franco y para rendir homenaje a quienes perecieron por ello. Así lo expresó en un escrito publicado en la revista Vértice al que sorprendentemente no se ha hecho alusión en los textos sobre la génesis del Valle de los Caídos y que, a nuestro juicio, debería considerarse parte de su simiente conceptual, si no al menos un inestimable precedente. Antes de adentrarnos en ello, esbozaremos un breve perfil intelectual de su autor.

De trayectoria profesional extensa y variada, entre la que se cuenta su faceta de dramaturgo, escritor y poeta, el contacto de Manuel Abril con las artes en los años previos al conflicto le convierte en uno de los críticos más destacados de nuestra plástica contemporánea. Casi una treintena de años después de su muerte, su colega Guillermo de Torre reivindicaba así su figura: "¿Quién recuerda hoy el nombre de Manuel Abril, autor de innumerables artículos donde seguía la actualidad artística de las décadas del 20 y del 30, autor asimismo de un libro en cuyas páginas se historia las artes plásticas del mismo período?". ${ }^{27}$

Su perfil como pensador se puede trazar a partir de sus palabras y de las opiniones vertidas en sus escritos, que vieron la luz en algunas de las publicaciones fundamentales en la renovación del arte español, tales como Revista de Occidente, Alfar, Arte (revista de la Sociedad de Artistas Ibéricos cuyo manifiesto firmó) o Cruz y Raya, esa "Revista de Occidente para señoritos bienpensantes" 28 considerada en alguna ocasión como de signo católico, ${ }^{29}$ de la que fue asiduo colaborador y parte de su comité editor. Por aquel entonces osaba hablar en

26 HUIDOBRO PARDO, Enrique; MOYA BLANCO, Luis; THOMAS, Manuel, 1943.

27 Como curiosidad, el texto se publicó justo después de producirse el fallecimiento del propio Guillermo de Torre, crítico de arte también él, entre otras cosas, quien arrancaba el opúsculo con las premonitorias palabras de "¡Triste destino póstumo el de los críticos de arte en España!". Véase TORRE, Guillermo de, 1971.

28 MAINER, José Carlos, 1996, p. 115-116.

${ }^{29}$ Cruz y Raya apareció en abril de 1933 y gozó de tres años de vida, coincidiendo prácticamente con el bienio cedista republicano. Su consideración como una revista politizada de signo católico se debe a las circunstancias que rodearon su fundación, propiciada por un grupo de católicos preocupados por la educación religiosa, y a la destacada importancia en sus páginas de la 
términos positivos del cubismo y de otras tendencias vanguardistas, y sus trabajos en el campo del teatro infantil le colocan entre los renovadores del género, que cultivó con éxito.

Desarrolló gran parte de su actividad profesional en una época políticamente convulsa, y a medida que nos adentramos en los años más decisivos en este sentido, podemos ubicar la posición ideológica de Abril. En 1931 se publicó su novela La salvación (Sociedad de Seguros del alma), en la que realizaba en clave humorística una apología de los valores católi$\cos ^{30}$ Durante la guerra civil permaneció en Madrid en una discreta posición. Se representaron algunas de sus obras teatrales escritas en los años anteriores y llegó a ofrecer alguna conferencia, como la que tuvo lugar el 17 de abril de 1938 en el marco de una jornada festiva de las artes organizada por CNT, y que llevó por título "Lo que debe ser el teatro". ${ }^{31}$

No sería hasta el ocaso de la contienda cuando retomó su actividad crítica. Desde entonces y hasta su fallecimiento en 1943, colaboró en algunas cabeceras como Arriba, Escorial, El Español, La Nueva España, Yugo o la revista Vértice que ahora comentamos, todas ellos vinculadas con Falange o con el régimen. Lógicamente, las circunstancias sociales de ese momento, con la superación de la guerra y la conformación del nuevo Estado, dieron rienda suelta a las simpatías de Abril hacia el pensamiento conservador, e intensificaron su visión del mundo desde una perspectiva cristiana. Hasta el punto incluso de llegar a hablar de adoctrinamiento a través de sus artículos, al menos en el ámbito escénico, ${ }^{32}$ pues el crítico defendía las virtudes de un teatro católico al escribir afirmaciones que abogaban por "un teatro que tuviera fe, esperanza y caridad como elementos rectores para interpretar la existencia". ${ }^{33}$

En cuanto a las artes plásticas, resulta especialmente interesante el artículo titulado "También el

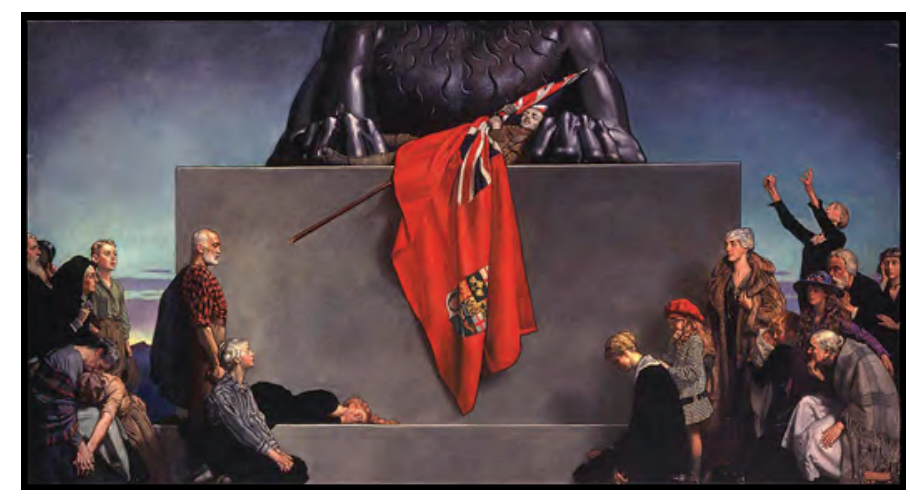

Fig. 4. John Byam Liston Shaw, La bandera, 1918. Canadian War Museum, Ottawa (Canadá).

arte es política" que Manuel Abril publicó en el diario Arriba en marzo de $1942 .{ }^{34}$ En él aborda la cuestión del arte de propaganda, solo que no en sentido explícito en cuanto a su contenido. Defiende que los artistas se mantengan al margen de la política, sin perjuicio de que sean promocionados por parte del Estado, incluso si esta promoción responde a fines políticos. Pero considera que las obras, su contenido, no tienen porqué ensalzar las glorias nacionales puesto que "el arte no necesita servir a otros fines que no sean los artísticos, sino que por el contrario, con solo el arte y la plástica engrandecen a su Patria". De manera natural, Abril encuentra la justificación a todo ello en el hecho de que el engrandecimiento de los pueblos se logra mediante "las actividades nobles de la criatura humana (...) [que] se reducen a las ramificaciones incontables de un solo y único fin: buscar a Dios en la verdad, en el arte y en la conducta". Y es que, como aclaran Díaz y Llorente, fue habitual en la alta posguerra la argumentación

figura de Miguel de Unamuno, persona de profunda aunque personal religiosidad. Al respecto de este asunto, puede consultarse GONZÁLEZ IZQUIERDO, Milagros, 1998.

${ }^{30}$ HERNÁNDEZ CANO, Eduardo. "Manuel Abril", Diccionario biográfico español de la Real Academia de la Historia (en línea). (Fecha de consulta: 2-2-2020).

31 "Festivales para mañana". La Libertad, 16-4-1938, p. 4.

32 CHECA PUERTA, Julio E. "Escena y política", Documentos para una historia del teatro español (en línea). (Fecha de consulta 9 4-2020).

33 La cita es de un artículo de Manuel Abril extraída del estudio mencionado en la nota anterior.

${ }^{34}$ ABRIL, Manuel, 1942, p. 3. Es necesario señalar que este artículo ha sido reproducido en diferentes estudios con fechas distintas: Julián Díaz y Ángel Llorente lo citan con la fecha que aquí se indica, mientras que Alina Navas lo enmarca en las postrimerías de la guerra, publicado el 25 de marzo de 1939. Creemos que la correcta es la primera, puesto que Abril hace referencia a la guerra del mundo y a la existencia de un escaparate de propaganda nazi en el centro de Madrid, y además el diario Arriba dejó de editarse durante la guerra civil y reapareció cuando entraron las tropas franquistas en Madrid, que no fue hasta el 28 de marzo, por lo que la fecha de 1939 debe ser un error. Véanse DíAZ SÁNCHEZ, Julián; LLORENTE HERNÁNDEZ, Ángel, 2004, p. 169 y NAVAS HERMOSILLA, Alina, 2016, p. 94. 
de que el arte verdadero era inseparable de la religión católica, y que aquel que se independizaba de esta perdía su valía estética. ${ }^{35}$

Habiéndonos aproximado al pensamiento de nuestro intelectual, se podrá inferir la importancia de las palabras que escribió en Vértice y que constituyen el epicentro de estas páginas. Con el rótulo "Dos monumentos posibles", ${ }^{36}$ el crítico de arte divagaba sobre cómo podría ser el monumento que reflejara el sentir que le embargaba en aquella decisiva fecha de abril de 1939, provocado por la victoria de Franco y el final de la guerra.

Parece oportuno reproducir las primeras líneas del texto para entender la profundidad de su emoción. "Hoy, cuando por vez primera, después de tres años de angustia, vuelven mi palabra y mi espíritu a comunicar con hermanos y a escribir en unas páginas que no manchan el alma del que lee, siento que no puedo comenzar, recomenzar mi vida de persona si no consagro la atención completa a los dos sentimientos primordiales que me impone el momento glorioso". El madrileño declaraba haber sufrido el dolor de su España desde el destierro -recordemos que permaneció en el Madrid republicano durante el conflicto armado- y decía volver a la vida "al entrar en relación y en comunicación con los míos". El júbilo que experimentaba le llevó a compartir estas palabras con la intención de "evocar las dos glorias esenciales que deben polarizar, de una manera exclusiva, el alma de todo español (...): la gloria del Más Allá; la gloria de nuestra España".

Manuel Abril proponía que esas dos glorias, los dos sentimientos que le afectaban, se concretaran a través del arte: "Pienso ahora que deberían existir en algún sitio dos monumentos análogos a estos dos que aquí presento. O uno solo, tal vez, uniendo ambos". Para dar claridad a ese único pensamiento que ocupaba su mente, dos imágenes ilustraban sus ideas, pues como él mismo explicaba, escogió el camino plástico y tendía a buscar todo aquello que entrase por los ojos.

La bandera [fig. 4], obra del británico John Byam Liston Shaw (1872-1919), custodiada en el Canadian War Museum (Ottawa, Canadá) y pintada en 1918 en honor a los soldados canadienses caídos en la Primera Guerra Mundial, se reproducía en la segunda y última página de su crónica. El cuadro representa a un grupo de personas que lloran el trágico pero noble final de un soldado fallecido, cuyo cuerpo inerte yace sobre un elevado podio al tiempo que sostiene el mástil de la antigua bandera canadiense que le cubre y cae dramáticamente, convirtiéndose en elemento central de la composición. Sobre el podio se alzan las patas de un descomunal león identificado con Gran Bretaña, bajo cuyo mando lucharon las tropas canadienses en aquel conflicto.

Abril percibe en la obra de Byam Shaw una "clara orientación que podría, realizada a la española, centrar la emoción en lo justo". La manera española aquí es clave, pues considera que la pintura es comedida en exceso y el pathos no está presente, por lo que sería necesario hacer de ella un cuadro vivo, en el que las emociones fueran reales y profundas. "Lo real sería entonces ideal", Ilegando a adquirir "gravedad de alegoría", sin duda una condición idónea para un monumento. Y según su punto de vista, esas emociones deberían estar perfectamente encasilladas en su personaje, que sería la digna encarnación de cada uno de los matices de una misma y común religión de patria: la fortaleza del hombre, la sorpresa y admiración por lo heroico en el niño, el sentimiento de abnegación de la mujer, la humilde devoción del pueblo... "Si existiera un monumento de esa hondura sentirían los hombres, desde niños, al mirarlo, la encarnación auténtica y real de la gloria de los caídos". Pues la finalidad del monumento no sería otra que la de ejemplarizar: "Las generaciones todas tendrían su ejemplo vivo ante los ojos...". Y entonces se pregunta: "¿Qué escultor habría en España capaz de realizar corpóreamente las figuras que hay en el cuadro (...)?". No llega a sugerir ningún nombre, ni siquiera el del artista -amigo suyo- con quien parece haber discutido el asunto y quien ve en ese óleo tan solo una sugerencia de cómo habría de ser el monumento...

El mismo amigo que concebía la fantasía monumental del crítico en clave cristiana. Y Abril, que entendía la creación desde una perspectiva sacra, aplaudía esa interpretación. Sería el anónimo artista quien pensó sustituir la figura del león por la de "una cruz de madera, lisa y sobria: una cruz gigantesca y colosal que pudiera mantener su dimensión, sin empequeñecerse, ante la inmensidad del firmamento". Como segundo ejemplo ilustrativo, la fotografía de un monumento levantado en Alemania -este sí, perfecto a ojos de Abril- ocupaba la mayor parte de la primera página del es-

${ }^{35}$ DÍAZ SÁNCHEZ, Julián; LLORENTE HERNÁNDEZ, Ángel, 2004, p. 173.

${ }^{36}$ ABRIL, Manuel, 1939. El texto entrecomillado de este párrafo y los siguientes, siempre que no se especifique otra cosa, ha sido extraído del artículo referido en esta nota. 
crito [fig. 5]. No se menciona cual es, pero se trata del desaparecido Monumento Nacional Schlageter en Düsseldorf, Alemania. Esta obra del arquitecto austríaco Clemens Holzmeister, proyectada en 1926 y levantada en 1931 en el cementerio norte de la ciudad alemana, se configuraba a partir de una serie de terrazas dispuestas concéntricamente, presididas en la zona central por una cruz de 27 metros de altura que apoyaba sobre un elevado pedestal pétreo. ${ }^{37}$ En eje con la cruz, en la terraza más profunda y de menor diámetro, se localizaba el acceso a una sala subterránea, oculta bajo el juego visual formado por los aros y curvas del pavimento. El espacio creado rezumaba un evidente sentido escénico, a modo de teatro griego, y su localización en una amplia zona de jardín que actuaba de natural apéndice al monumento, le infería un carácter público, como de plaza, que invitaba a la congregación de las masas [fig. 6].

El monumento se construyó en honor a Albert Leo Schlageter, activista alemán ejecutado por los franceses por espionaje y sabotaje durante la ocupación franco-belga de la zona del Ruhr, advenida como reacción al incumplimiento de las condiciones establecidas en el Tratado de Versalles por parte de la República de Weimar. Tras su muerte en 1923, acaecida precisamente en el lugar que ocupó el memorial al que nos referimos, grupos de extrema derecha -incluido el Partido Nazi- idealizaron la figura del condenado hasta el punto de considerarle un mártir, y crearon un culto para venerar al que denominaron como el primer soldado caído del III Reich. ${ }^{38}$ Alrededor de un centenar de monumentos se alzaron en su nombre, siendo el de Düsseldorf el más importante, constituido en monumento nacional. Fue demolido en 1946 por orden municipal. ${ }^{39}$

Llama la atención que Manuel Abril no indicara siquiera de qué lugar específico se trataba, habida cuenta de la afinidad entre relato como hito nacionalista alemán y el planteamiento que él mismo sostenía para el posible monumento de la patria española. En cambio, se recreó en elogiar la superioridad simbólica y plástica de ese signo supremo, puro y sublime con el que identificaba la cruz $^{40}$ definiéndolo como "esfinge cristiana", "proporción milagrosa de dos rectas desnudas y castas", "equilibrio de infinito" o "señal que mar-

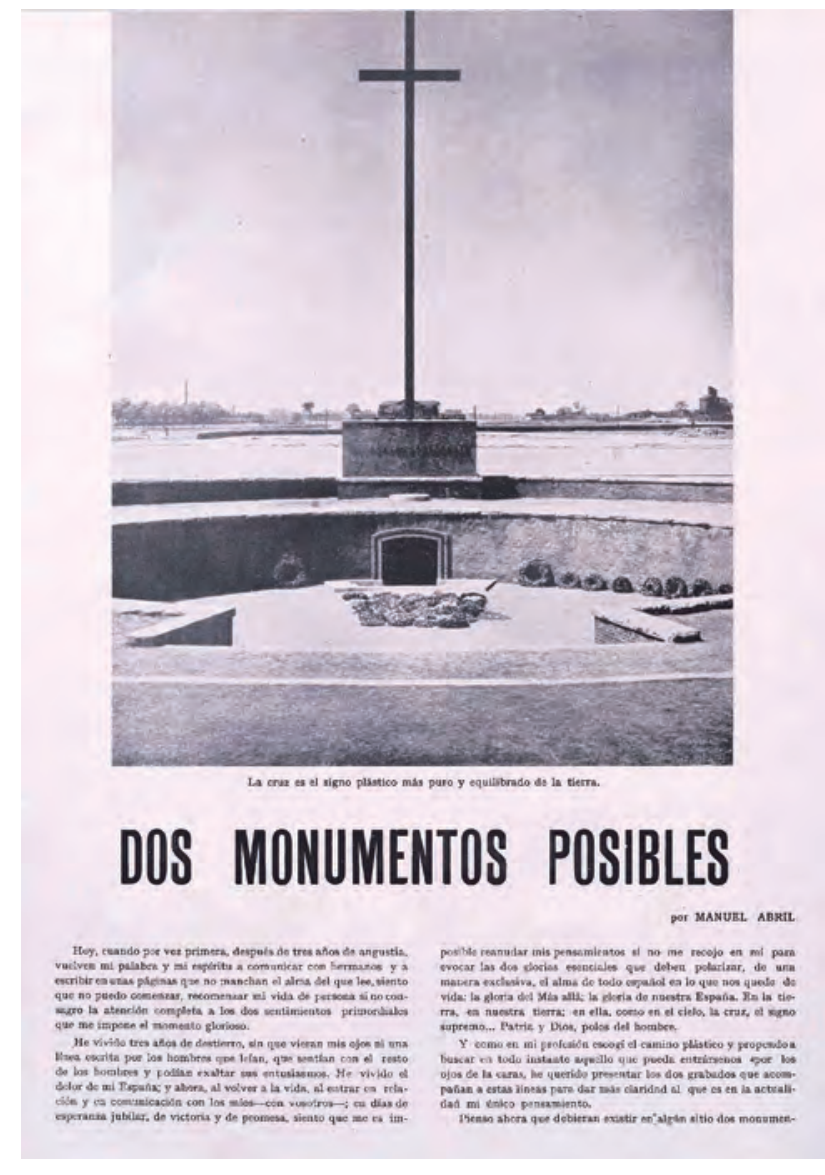

Fig. 5. Monumento alemán ilustrando el artículo de Manuel Abril “Dos monumentos posibles", publicado en el núm. 21 de la revista Vértice (abril de 1939).

ca en el cielo los puntos cardinales del espíritu: las dos dimensiones supremas -vertical y horizontal: autoridad y amor: brazos abiertos-".

En suma, lo que nuestro crítico planteaba era un monumento a la religión de la patria que combinase la heroicidad de los caídos y los profundos sentimientos del pueblo como ofrenda a ellos, junto con la cruz que es "norma del sacrificio y del dolor" pero también "redención y sentido superior del verdadero y hondo patriotismo".

37 KNAUFF, Michael, 1995, p. 178.

38 MOSSE, George L., 2016, p. 232.

39 Tras la victoria aliada de la Segunda Guerra Mundial se derribó el monumento sobre el que se había proyectado un culto de exacerbado frenesí y con el que se asociaban valores propios del nacionalsocialismo. En la actualidad quedan en pie pocos de los memoriales dedicados al inmortal soldado alemán. KNAUFF, Michael, 1995, p. 168, 172.

40 La sola mención que hemos hallado al artículo de Manuel Abril se refiere precisamente a su defensa de la cruz en relación a la consagración de este símbolo como elemento principal de los monumentos a los caídos. LLORENTE HERNÁNDEZ, Ángel 1992, p. 175. 


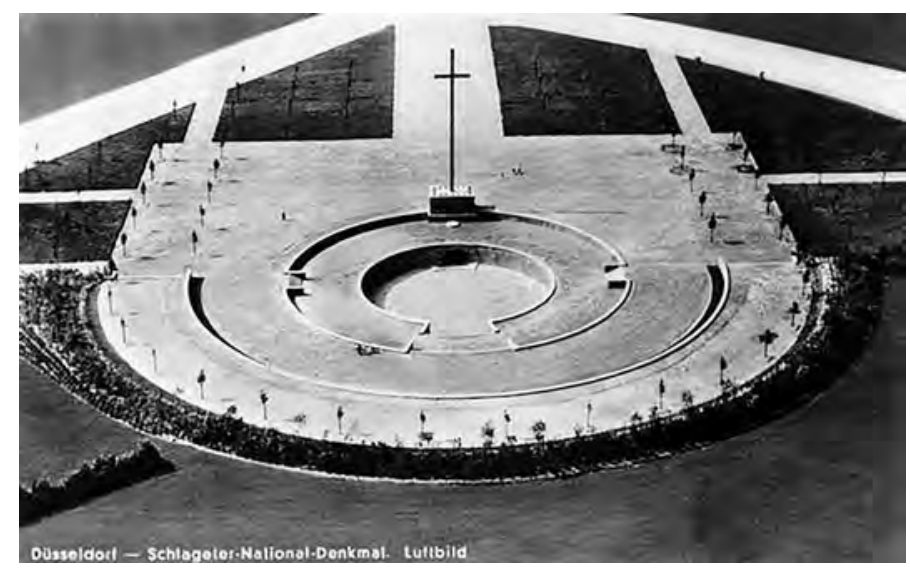

Fig. 6. Monumento Nacional Schlageter, 1931, demolido en 1946. Düsseldorf (Alemania).

\section{Conclusiones: "Patria y Dios, polos del hombre"}

En el presente artículo nos hemos dedicado a apuntar y analizar las concomitancias formales y conceptuales encontradas entre el Valle de los Caídos y el monumento pensado por Manuel Abril en 1939, incidiendo especialmente en sus significados y poniéndolos en relación con las circunstancias históricas que motivaron su advenimiento. Esa coincidente significación de naturaleza estratégica y política, que fue de gran relevancia para el gobierno franquista, hoy se relaciona irremediablemente con la construcción del sitio de Cuelgamuros, pues allí se vertieron las aspiraciones megalómanas del caudillo invicto. Se perseguía la gloria eterna de los vencedores, pero no solo en el Valle sino también en los numerosos hitos conmemorativos levantados durante la posguerra como parte de la política dictatorial, construida sobre los valores católicos de los que se apropió, y sustentada por una arquitectura puesta al servicio del Estado.

Manuel Abril supo imaginar un hito que aunara todo lo que años más tarde entrañaría el Valle de los Caídos, exponiendo con bastante detalle cómo debía ser un monumento que, no solo por sus aspiraciones sino también por sus características, alcanzaba el rango de nacional. Y lo hizo en fecha temprana. Sus sugerencias aparecieron al tiempo en que se anunciaba la idea de crear un "magno monumento" patriótico, como se decía en aquel decreto de 1939. Siguiendo su propio impulso emocionado, esbozó las cualidades fundamentales del que se convertiría veinte años más tarde en verdadero Monumento Nacional.
Es preciso subrayar fundamentalmente dos aspectos de su propuesta de monumento que le convierten en precedente incuestionable del Valle de los Caídos. En primer lugar, la intención aleccionadora del conjunto para las generaciones futuras, lo que también se indica explícitamente en los decretos relativos al Valle, y en segunda instancia, la exaltación de la cruz. Sobre el primero conviene señalar que ese poder ejemplarizante es diferente en ambos casos, pues en el monumento de Abril se conseguiría mediante la empatía, con la representación figurativa de unos tipos patrióticos en los que la ciudadanía se pudiera ver reflejada -y al hacerlo se identificara con ellos, sintiendo la misma emoción que emanaría de las estatuas "vivas"-, mientras que en el Monumento Nacional, el impacto emocional se logra por medio del empequeñecimiento del visitante, aplastado por la descomunal construcción, tanto como la gesta por la que fue concebido y que quiso vanagloriar.

Respecto al segundo aspecto -el elogio de la cruzañadiremos que, probablemente, hablar de Abril como visionario sería excesivo, teniendo en cuenta lo conocido sobre el predominio de la cruz en las acciones conmemorativas iniciadas durante la guerra, y la presencia extendida de cruces en múltiples memoriales que homenajean a los caídos en todo el mundo. Pero sus aportaciones sobre el sentido plástico del signo, como la apuesta por el gigantismo para contrarrestar la mengua de su perfil recortándose sobre la inmensidad del firmamento, o la sobriedad de sus palos, son dos de los distintivos de la poderosa cruz que hoy domina el valle en la sierra de Guadarrama -la del arquitecto Diego Méndez, aunque cualquiera de las planteadas en el concurso de anteproyectos lo era en mayor o menor medida-. Pero no solo en cuanto a estética fue un claro antecedente, también fue acertada su interpretación de la cruz como símbolo supremo de redención y patriotismo.

Asimismo, es importante señalar los puntos de conexión existentes entre el Monumento Nacional Schlageter y el Valle de los Caídos. Puede que la elección del monumento alemán por parte de Abril como ejemplo inspirador fuera casual, pero en absoluto fue baladí. Más allá de las semejanzas formales -salvando las distancias en cuanto a proporciones- y de su advocación a los caídos, es posible establecer otras correspondencias relevantes. Por ejemplo, la condición subterránea del espacio interior de ambas construcciones. En los dos casos estos espacios fueron pensados como criptas, y aunque en el alemán no se conservaron restos mortales sí se dispuso un cenotafio. Fueron, por tanto, lugares ideados para alojar enterramientos (no olvidemos 
que el de Schlageter se alzó en las inmediaciones de donde fue ejecutado) de nobles caídos por la patria, mártires cuyo ejemplo justificaba la graduación de los monumentos como nacionales.

Además, el propósito aleccionador que antes comentábamos también se desprendía del memorial Schlageter, de hecho, hubo una firme intención por parte del Comité del Monumento de adoctrinar al pueblo alemán y sobre todo a la juventud, inculcándoles el sentimiento de autoafirmación nacional. ${ }^{41}$ Un sentimiento que surgía de las desgracias y calamidades sufridas por el pueblo alemán desde la I Guerra Mundial, y que se presentaba como único camino hacia la resurrección de Alemania. Como símbolo de ese renacimiento, la cruz que, probablemente, al decir de Michael Knauff, fue elegida de forma deliberada. ${ }^{42}$ Un signo que representaba un punto de inflexión en la historia del pueblo alemán y la unión nacional, así como el verdadero sentido del cristianismo, doctrina que el nacionalsocialismo -igual que el franquismo- empleó para narrar su particular cruzada y enfatizar la naturaleza sagrada de la nación. ${ }^{43}$

Estas premisas sobre las que se sustenta el sólido paralelismo de los casos germano e hispano, expuesto aquí en relación a la construcción del Valle de los Caídos, apuntan a la rotunda conclusión que puede expresarse a través de las palabras de nuestro lúcido crítico: "Patria y Dios, polos del hombre", aseveraba en su artículo. ${ }^{44} \mathrm{Y}$ con ello vinculaba inexorablemente religión y Estado. Una unión indisociable que, como hemos visto, se materializó en el monumento del Valle de los Caídos, y a la postre constituyó, no lo olvidemos, la esencia de la política nacionalcatólica.

Por todo lo aquí argumentado, consideramos que la oda a la religión de la patria que fue la propuesta de Manuel Abril publicada en "Dos monumentos posibles", debe entenderse como un digno precedente del Monumento Nacional, tanto a nivel conceptual como formal. Se trata, igualmente, de poner en valor el artículo sin dejar de notar a su insigne autor, un destacado intelectual cuyas aportaciones al mundo de las artes no fueron insignificantes, como hemos podido comprobar. Por ese motivo apreciamos especialmente su idea de monumento, y creemos que en su época también debió de ser tenida en consideración. Es más, son tales las coincidencias halladas entre su proyecto imaginado y el le- vantado en el Valle de los Caídos, y tan oportuna su aparición, coincidiendo con el anuncio de creación del grandioso memorial, que no es posible descartar que su plan orientara de alguna manera los cauces por donde fluyeron las ideas y características que finalmente se concretaron en la sierra madrileña.

\section{Bibliografía}

ABRIL, Manuel. "Dos monumentos posibles". Vértice, 21, abril de 1939, s.p.

ABRIL, Manuel. "También el arte es política". Arriba, 253-1942, p. 3.

BONET CORREA, Antonio. "Espacios arquitectónicos para un nuevo orden". En: BONET CORREA, A. (coord.). Arte del franquismo. Madrid: Cátedra, 1981, p. 11-46.

CHECA PUERTA, Julio E. "Escena y política", Documentos para una historia del teatro español (en línea). En: $<$ http://teatro.es/contenidos/documentosParaLaHisto $\mathrm{ria} / \mathrm{Docs} 1941 /$ texto.php?pag $=3 \&$ anio $=1941 \& \mathrm{sec}=1 \&$ sub $=2>$ (Fecha de consulta 9-4-2020).

DÍAZ SÁNCHEZ, Julián; LLORENTE HERNÁNDEZ, Ángel. La crítica de arte en España (1939-1976). Madrid: Akal, 2004.

España. Orden de 7 de agosto de 1939 disponiendo queden supeditados a la aprobación de este Ministerio toda iniciativa de monumentos en general. Boletín Oficial del Estado, 22-8-1939, núm. 234, p. 4614.

España. Decreto de 1 de abril de 1940 disponiendo se alcen Basílica, Monasterio y Cuartel de Juventudes, en la finca situada en las vertientes de la Sierra del Guadarrama (El Escorial), conocida por Cuelga-muros, para perpetuar la memoria de los caídos en nuestra Gloriosa Cruzada. Boletín Oficial del Estado, 2-4-1940, núm. 93 , p. 2240.

España. Decreto-ley de 23 de agosto de 1957 por el que se establece la Fundación de la Santa Cruz del Valle de los Caídos. Boletín Oficial del Estado, 5 de septiembre de 1957, núm. 226, p. 834-835.

"Festivales para mañana". La Libertad, 16-4-1938, p. 4

GARCÍA-GUTIÉRREZ MOSTEIRO, Javier. "La depuración profesional de arquitectos tras la guerra civil: un punto de inflexión en la trayectoria de la arquitectura en España". En: CABAÑAS BRAVO, M.; LÓPEZ-YARTO ELIZALDE, A.; RINCÓN GARCÍA, W. (coords.). Arte en tiempos de guerra. Madrid: CSIC, 2008, p. 355-365.

GUERRERO, E. "En honor de los caídos por Dios y por España". Razón y Fe, 1958, 158, 726-727, p. 13-22.

GONZÁLEZ IZQUIERDO, Milagros. "Cruz y Raya: Manue de Falla y Miguel de Unamuno". Revista de Filología de la Universidad de La Laguna, 1998, 16, p. 83-102.

HERNÁNDEZ CANO, Eduardo. "Manuel Abril", Diccionario biográfico español de la Real Academia de la Historia (en línea). En: <http://dbe.rah.es/biografias/4720/ manuel-abril> (Fecha de consulta: 2-2-2020).

HUIDOBRO PARDO, Enrique; MOYA BLANCO, Luis; THOMAS, Manuel. "Concurso de anteproyectos para una gran cruz monumental convocado por el Patronato del Monumento Nacional a los Caídos: Primer Premio". Revista Nacional de Arquitectura, 1943, 18-19, p. $247-253$.

\footnotetext{
${ }^{41}$ KNAUFF, Michael, 1995, p. 183.

42 KNAUFF, Michael, 1995, p. 183.

43 MOSSE, George L., 2016, p. 137.

${ }^{44}$ ABRIL, Manuel, 1939.
} 
JIMÉNEZ-BLANCO, Ma Dolores (com.). Campo cerrado. Arte $y$ poder en la posguerra española, 1939-1953. Madrid: Museo Nacional Centro de Arte Reina Sofía, 2016.

KNAUFF, Michael. "Das Schlageter-Nationaldenkmal auf der Golzheimer Heide in Düsseldorf". Geschichte im Westen, 1995, 2, año 10, p. 168-191.

LAVIADA, Manuel; MOYA, Luis; SERRANO, Gonzalo. "Sueño arquitectónico para una exaltación nacional". Vértice, 36, septiembre de 1940, p. 7-11.

LLORENTE HERNÁNDEZ, Ángel. Arte e ideología en la España de la Postguerra (1939-1951), 2 vols. Madrid: Editorial de la Universidad Complutense, 1992.

LLORENTE HERNÁNDEZ, Ángel. Arte e ideología del franquismo. Madrid: Visor, 1995

MAINER, José Carlos. "Contra el Marasmo: Las Revistas Culturales en España (1900-1936)". En: CARMONA, E.; LAHUERTA, J.J. (coms.). Arte moderno y revistas españolas 1898-1936. Madrid: Ministerio de Eduación y Cultura, 1996, p. 103-116.

MOSSE, George L. Soldados caídos. La transformación de la memoria de las guerras mundiales. Zaragoza: Prensas de la Universidad de Zaragoza, 2016.

NAVAS HERMOSILLA, Alina. "Italia, entre lo clásico y lo moderno". En: JIMÉNEZ-BLANCO, M.D. (com.). Campo cerrado. Arte y poder en la posguerra española. 19391953. Madrid: Museo Nacional Centro de Arte Reina Sofía, 2016, p. 92-95.

OLMEDA NICOLÁS, Fernando. El Valle de los Caídos. Una memoria de España. Barcelona: Península, 2009.

SEVILLANO-CALERO, Francisco. "Caídos por Dios y por España. El culto a la muerte en la fundación de la dictadura franquista". Historia Contemporánea, 2017, 55, p. 609-635. DOI: $10.1387 /$ hc. 18095.

SUEIRO, Daniel. La verdadera historia del Valle de los Caídos. Madrid: Sedmay, 1976.

TORRE, Guillermo de, "El crítico Manuel Abril", Bellas Artes, 1971, 7, p. 5-6.

VÁZQUEZ ASTORGA, Mónica. "Los monumentos a los caídos: ¿un patrimonio para la memoria o para el olvido?". Anales de Historia del Arte, 2006, 16, p. 285-314. 